Sādhanā Vol. 39, Part 5, October 2014, pp. 1151-1164. (C) Indian Academy of Sciences

\title{
Bond graph to digraph conversion: A sensor placement optimization for fault detection and isolation by a structural approach
}

\author{
ALEM SAÏD* and BENAZZOUZ DJAMEL \\ Solid Mechanics and Systems Laboratory (LMSS), University M'Hamed Bougara \\ Boumerdes, Boumerdes 35000, Algeria \\ e-mail: alem-said@umbb.dz; dbenazzouz@umbb.dz
}

MS received 22 March 2012; revised 7 October 2013; accepted 6 January 2014

\begin{abstract}
In this paper, we consider the optimal sensors placement problem for faults detection and isolation using a novel structural and qualitative approach. This approach is based on the conversion of Bond Graph to Digraph representation of a structural system. When the fault detection and isolation of an existing system's sensors are impossible or uncertain, a reconfiguration sensor placement of this system should be reconsidered. This paper proposes how this reconfiguration takes place by recovering all missing or redundant parts of the system. This novel approach is illustrated over a thermo-fluid application.
\end{abstract}

Keywords. Fault detection and isolation; sensor placement optimization; digraph; bond graph; structural approach.

\section{Introduction}

A structural approach was introduced for systems with unknown parameters in the absence of quantitative or incorrect values due to measurement errors, this last aspect being unavoidable in modelling physical processes. Thus, it is a matter of a maximum exploitation of the structural approach behind any computation. The method is based on the graph theory. While constructing the structural graph (or structural matrix) of the system, some information maybe lost. This is due to the fact that the construction is generally done from the state equations, which do not explicitly need the use of all constitutive relations. In our case, we do not need all the constitutive relations of the studied system. The Bond Graph (BG) tool defined in Paynter (1961) formalized in Kamopp \& Rosenberg (1975) and Breedveld (1984), is a network type language, which represents graphically, with a single language, many physical systems. It is placed like an intermediate stage between the physical description of a dynamic system and the construction of a mathematical model phase, by graphical representation of the powers exchanges between various components of the system. The modelling of a physical system with BG does not require the

*For correspondence 
writing of general laws of energy conservation. It is based, principally, on the characterization of the power exchanges phenomena within the system.

In the first part of the paper, we present some definitions on linear structured systems, by pointing out the interpretation of the structural properties through the association of Digraph. A method of solving the sensor placement problem for faults detection and isolation in the system will be presented. The analogy between Digraph and BG is presented over an example of a two tank system given in the second part. The novel approach to optimize the sensor placement using the conversion from BG to Digraph is presented. An industrial water heater thermo-fluid system is taken as a case study.

\section{Sensor placement and structural problem formulation}

\subsection{Linear structured systems and the associated digraph}

In this part, we present some general definitions and results on linear structured systems (Commault \& Dion 2003; Commault et al 1991; Dion \& Commault 1993).

We consider linear systems as given in Eq. (1) with parameterized entries denoted by $\Sigma_{\Lambda}$

$$
\sum_{\wedge}\left\{\begin{array}{c}
\dot{x}(t)=A x(t)+L f(t) \\
y(t)=C x(t)+M f(t) .
\end{array}\right.
$$

Eq. (1) is called a linear structured system if the entries of the composite matrix $\mathrm{J}$ fixed zeros or independent parameters (no algebraic relationship between parameters). $\Lambda=\left\{\lambda_{1}, \lambda_{2} \ldots, \lambda_{k}\right\}$ denotes the set of independent parameters of the composite matrix $\mathrm{J}$.

For the sake of simplicity, the dependence of the system matrices on $\Lambda$ will not be made explicit in the notation. A structured system represents a large class of parameter dependent linear systems.

The structure is given by the location of the fixed zero entries of J. This structure often comes from physical particularities of the system (for example, interconnection of subsystems); thus the only exact knowledge on the system is the structure, i.e., the absence of direct relations between variables as state variables for example.

For such systems one can study generic properties i.e., properties which are true for almost all values of the parameters collected in $\Lambda$.

More precisely, a property is said to be generic (or structural) if it is true for all values of the parameters (i.e., any $\Lambda \in \mathrm{R}^{k}$ ) outside a proper algebraic variety of the parameter space, i.e., the zero set of a finite number of non-trivial polynomials in the parameters. That means if it depends only on the type of elements which composes this system and on the way of which they are interconnected, not on numerical value of its parameters. Or if it is checked for all the values of the parameters except possibly for some particular values.

A directed graph $\mathrm{G}\left(\Sigma_{\Lambda}\right)=(\mathrm{Z}, \mathrm{W})$ can be associated to the structured system $\Sigma_{\Lambda}$ of type of Eq. (1).

Where the matrix $J=\left(\begin{array}{cc}A & L \\ C & M\end{array}\right)$ is structured.

The vertex set is $\mathrm{Z}=\mathrm{F} \mathrm{UX} \mathrm{U} \mathrm{Y}$ where $\mathrm{F}, \mathrm{X}$ and $\mathrm{Y}$ are the fault, state and output sets given by $\left\{\mathrm{f}_{1}, \mathrm{f}_{2} \ldots, \mathrm{f}_{r}\right\},\left\{\mathrm{x}_{1}, \mathrm{x}_{2} \ldots, \mathrm{x}_{n}\right\}$ and $\left\{\mathrm{y}_{1}, \mathrm{y}_{2} \ldots, \mathrm{y}_{p}\right\}$ respectively,

The arc set is $\mathrm{W}=\left\{\left(\mathrm{f}_{i}, \mathrm{x}_{j}\right) \mid \mathrm{L}_{j i} \neq 0\right\} \mathrm{U}\left\{\left(\mathrm{x}_{i}, \mathrm{x}_{j}\right) \mid \mathrm{A}_{j i} \neq 0\right\} \mathrm{U}\left\{\left(\mathrm{x}_{i}, \mathrm{y}_{j}\right) \mid \mathrm{C}_{j i} \neq 0\right\} \mathrm{U}\left\{\left(\mathrm{f}_{i}, \mathrm{y}_{j}\right) \mid\right.$ $\left.\mathbf{M}_{j i} \neq 0\right\}$, where $\mathrm{A}_{j i}\left(\right.$ resp. $\mathrm{C}_{j i}, \mathrm{~L}_{j i}, \mathrm{M}_{j i}$ ) denotes the entry $(\mathrm{j}, \mathrm{i})$ of the matrix A(resp. C ,L,M).

Moreover, recall that a directed path in $\mathrm{G}\left(\Sigma_{\Lambda}\right)$ from a vertex $\mathrm{i}_{\mu 0}$ to a vertex $\mathrm{i}_{\mu q}$ is a sequence of $\operatorname{arcs}\left(i_{\mu 0}, i_{\mu 1}\right),\left(i_{\mu 1}, i_{\mu 2}\right) \ldots,\left(i_{\mu q-2}, i_{\mu q-1}\right),\left(i_{\mu q-1}, i_{\mu q}\right)$ such that $i_{\mu t} U Z$ for $t=0,1 \ldots, q$ and 
$\left(\mathrm{i}_{\mu t-1}, \mathrm{i}_{\mu t}\right) \mathrm{U} \mathrm{W}$ for $\mathrm{t}=1,2 \ldots, \mathrm{q}$. The length of a path is the number of its arcs, each arc being counted to the number of times it appears in the sequence. For the last sequence, the path has length q.

Occasionally, we denote the path $\mathrm{P}$ by the sequence of vertices, it consists of $\mathrm{P}=\left(\mathrm{i}_{\mu 0}, \mathrm{i}_{\mu 1} \ldots\right.$, $\left.\mathrm{i}_{\mu q-1}, \mathrm{i}_{\mu q}\right)$.

Moreover, if $\mathrm{i}_{\mu 0} \in \mathrm{F}$ and, $\mathrm{i}_{\mu q} \in \mathrm{Y}, \mathrm{P}$ is called a fault-output path. A path which is such that $\mathrm{i}_{\mu 0}=\mathrm{i}_{\mu q}$ is called a circuit.

A set of paths with no common vertex is said to be a vertex disjoint. A k-linking is a set of $\mathrm{k}$ vertex disjoint fault-output paths; it is also called a linking of size $\mathrm{k}$. A linking is maximal when $\mathrm{k}$ is maximal.

Using their associated graphs many important results have been obtained for these systems on structural controllability, decoupling, disturbance rejection and so on (Commault et al 1991; Dion \& Commault 1993; Lin 1974).

As a first example of these results, the graph characterization of the structural observability was given by Commault (Commault \& Dion 2003; Commault et al 1991; Dion \& Commault 1993) and Lin (1974).

Let $\Sigma_{\Lambda}$ be the linear structured system defined by Eq. (1) with its associated graph $\mathrm{G}\left(\Sigma_{\Lambda}\right)$. The system (in fact the pair $(\mathrm{C}, \mathrm{A})$ ) is structurally observable if and only if:

There exists a state-output path starting from any state vertex in X,

There exist a set of vertex disjoint circuits and state-output paths which cover all state vertices.

Now, if we consider system $\Sigma_{\Lambda}$ given by Eq. (1) where its transfer matrix is $\mathrm{T}_{\Lambda}(\mathrm{s})=$ $\mathrm{C}(\mathrm{sI}-\mathrm{A})^{-1} \mathrm{~L}+\mathrm{M}$, thus, we can calculate the generic rank of $\mathrm{T}_{\Lambda}(\mathrm{s})$ using the result given by Commault (Commault \& Dion 2003; Commault et al 1991). Therefore, the generic rank of $\mathrm{T} \Lambda(\mathrm{s})$ is equal to the size of a maximal linking in $\mathrm{G}\left(\Sigma_{\Lambda}\right)$.

Example 1: To illustrate this linear structure system, let us consider $\Sigma \Lambda$ of type (7), with three faults and two outputs. Thus the obtained four matrices are as follows:

$$
\begin{aligned}
& A=\left[\begin{array}{lll}
0 & 0 & 0 \\
\lambda_{1} & 0 & 0
\end{array}\right], L=\left[\begin{array}{ccc}
\lambda_{3} & 0 & 0 \\
0 & \lambda_{4} & 0
\end{array}\right] \\
& C=\left[\begin{array}{ccc}
0 & \lambda_{6} & 0 \\
0 & 0 & \lambda_{7}
\end{array}\right], M=\left[\begin{array}{lll}
0 & 0 & 0 \\
0 & 0 & 0
\end{array}\right] .
\end{aligned}
$$

The entries of these matrices are the free parameters $\Lambda=\left(\lambda_{1}, \lambda_{2} \ldots, \lambda_{7}\right)$. The obtained associated graph $\mathrm{G}\left(\Sigma_{\Lambda}\right)$ is represented in figure 1 .

We can see that, this system is structurally observable as it has been defined. Indeed, there is a state-output path starting from any state vertex and the set of vertex disjoint paths $\left(\mathrm{x}_{1}, \mathrm{x}_{2}, \mathrm{y}_{1}\right)$ and $\left(\mathrm{x}_{3}, \mathrm{y}_{2}\right)$ covers all state vertices. The system has clearly rank two since there exist a linking of size two in the graph given in figure 1.

\subsection{Solving the FDI problem by additional sensor placement}

In general, the above defined FDI problem has no solution using only the existing sensors of the system. In such a case, we can add new sensors on the system. Let us define the new output vector $\mathrm{z}$ which collects the potential new measurements as

$$
z(t)=D x(t)+N f(t) .
$$




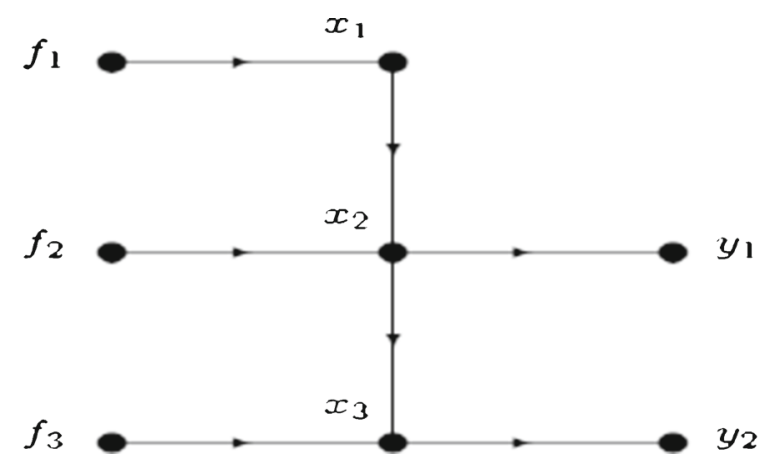

Figure 1. Graph $\mathrm{G}(\Sigma \Lambda)$.

All the previous definitions can be extended to the composite structured system $\Sigma_{c \Lambda}$ with the associated graph $\mathrm{G}\left(\Sigma_{c \Lambda}\right)$, where $\Sigma_{c \Lambda}$ is defined as follows

$$
\sum_{C \wedge}\left\{\begin{array}{l}
\dot{x}(t)=A x(t)+L f(t) \\
y(t)=C x(t)+M f(t) \\
z(t)=D x(t)+N f(t) .
\end{array}\right.
$$

$F_{1}$ defines the set of faults vertices, $Y_{1}$ is the set of output vertices and $\mathrm{X}_{1}$ the set of state vertices in any fault-output path from $\mathrm{F}_{1}$ to $\mathrm{Y}_{1}$.

Now, let us consider the new structured system defined by its graph with the input set $F_{1}$ and the output set $\mathrm{Y}_{1}$, the state set $\mathrm{X}_{1}$, the set of edges correspond to the edges in any path from $\mathrm{F}_{1}$ to $\mathrm{Y}_{1}$. This corresponding structured system is denoted by $\Sigma_{1 \Lambda}$ and its graph by $\mathrm{G}\left(\Sigma_{c \Lambda}\right)$.

The directed graph $\mathrm{G}\left(\Sigma_{c \Lambda}\right)$ can be associated with the structured system $\Sigma_{c \Lambda}$ of type (3). In fact $\mathrm{G}\left(\Sigma_{c \Lambda}\right)$ is obtained from $\mathrm{G}\left(\Sigma_{\Lambda}\right)$ by adding $\mathrm{q}$ output vertices $\mathrm{z}_{1}, \ldots, \mathrm{z}_{q}$ and edges from $\mathrm{F} \cup \mathrm{X}$ to $\mathrm{Z}$ in $\mathrm{G}\left(\Sigma_{\Lambda}\right)$ corresponding to non-null parameters of matrices $\mathrm{D}$ and $\mathrm{N}$.

The variable $\mathrm{w}_{i}$ is said to be measurable with an additional sensors if we can add a new sensor with corresponding output $\mathrm{z}_{j}$ such that in the graph of the composite system $\mathrm{G}\left(\Sigma_{c \Lambda}\right)$ there is an edge $\left(\mathrm{w}_{i}, \mathrm{z}_{j}\right)$ between this variable and the new output. Let us denote by $\mathrm{W}_{m}$ the set of measurable fault and state variables (Commault \& Dion 2003; Alem \& Benazzouz 2011).

Now consider a linear structured system $\Sigma_{\Lambda}$ with graph $\mathrm{G}\left(\Sigma_{\Lambda}\right)$ and suppose that the FDI problem without additional sensors has no solution, thus the FDI problem with additional sensors has a solution only if

$$
(F 1 U X 1) \cap W m \neq \Phi .
$$

Since the FDI problem without additional sensors has no solution, we have $\mathrm{F}_{1} \neq \phi$. If the condition of Eq. (4) is not satisfied, there is no edge connecting $F_{1} \cup X_{1}$ to the additional vertices of $\mathrm{Z}$, thus the FDI problem with additional sensors has no solution. To check if supplementary measurements allow to solve the problem we could simply add the new output vertices, the new edges, and verify on the modified graph that the condition is satisfied.

From directed graph $\mathrm{G}\left(\Sigma \mathrm{c}_{\Lambda}\right)$ we can note that the new sensor $\mathrm{z}_{1}$ is linked to the state $\mathrm{x}_{1}$ and the outputs $y_{1}$ and $y_{2}$ are linked to the other states, that means there is a linking of size 3 from $F_{1}$ to $\left(Y_{1} \cup Z\right)$, namely $\left(f_{1}, x_{1}, z_{1}\right),\left(f_{2}, x_{2}, y_{1}\right)$ and $\left(f_{3}, x_{3}, y_{2}\right)$. Thus the additional sensor placement solves the FDI problem. 


\section{Bond graph to digraph conversion}

Few authors established a comparison between Bond Graphs (BG) and the linear graphs from points of view of modelling and mathematical formulation (Bell \& Martens 1974; Ort \& Martens 1974; Perelson \& Oster 1976). In addition, they proposed the conversion of BG into a linear graph. The conversion of BG starting from the Matroïdes was proposed by Birkett (1990). Recently, Rahmani (Rahmani et al 1997) proposed a new concept relating to BG, entitled family of causal cycles like equivalence with the concept of family of cycles in a Digraph.

In this paper, we propose a new approach for the conversion from the BG to Digraph; this approach is generally used to draw the Digraph (König 1931), this will be used for our case study of the linear structured system associated to Digraph (Commault \& Dion 2003; Alem \& Benazzouz 2011). Let us consider the two tanks system (Benazzouz et al 2009; Samantaray \& Ould Bouamama 2008) given in figure 2.

Figure 2 shows the system of two tanks and its corresponding BG model which are denoted by $\mathrm{C}_{1}$ and $\mathrm{C}_{2} . \mathrm{R}_{1}$ and $\mathrm{R}_{2}$ are two controlled valves, $\mathrm{Ms}_{f}$ is the pump, $\mathrm{u}_{1}$ and $\mathrm{u}_{2}$ are command, $\mathrm{De}_{1}, \mathrm{De}_{2}$ and $\mathrm{Df}_{1}$ are detectors used for detecting any faults in monitoring the system. $\mathrm{r}_{1}, \mathrm{r}_{2} \ldots$ $\mathrm{r}_{6}$ are the residues or the faults indicators (Benazzouz et al 2009; Mellal et al 2011).

Table 1 represents the faults signature matrix that contains in rows the Bond graph elements and in columns residues or faults indicators. $\mathbf{M}_{b}$ denotes the structural monitorability/detectability of faults, if $\mathbf{M}_{b}=1$ the fault is detectable on the system element (a residue in its line contains at least one non-zero value), in this case study all the faults are detectable, which means that we have a maximal faults detection. $\mathrm{I}_{b}$ is the structural isolability of the detectable fault, if $\mathrm{I}_{b}=1$ the fault is isolable from the other faults, that means the two tested residues have

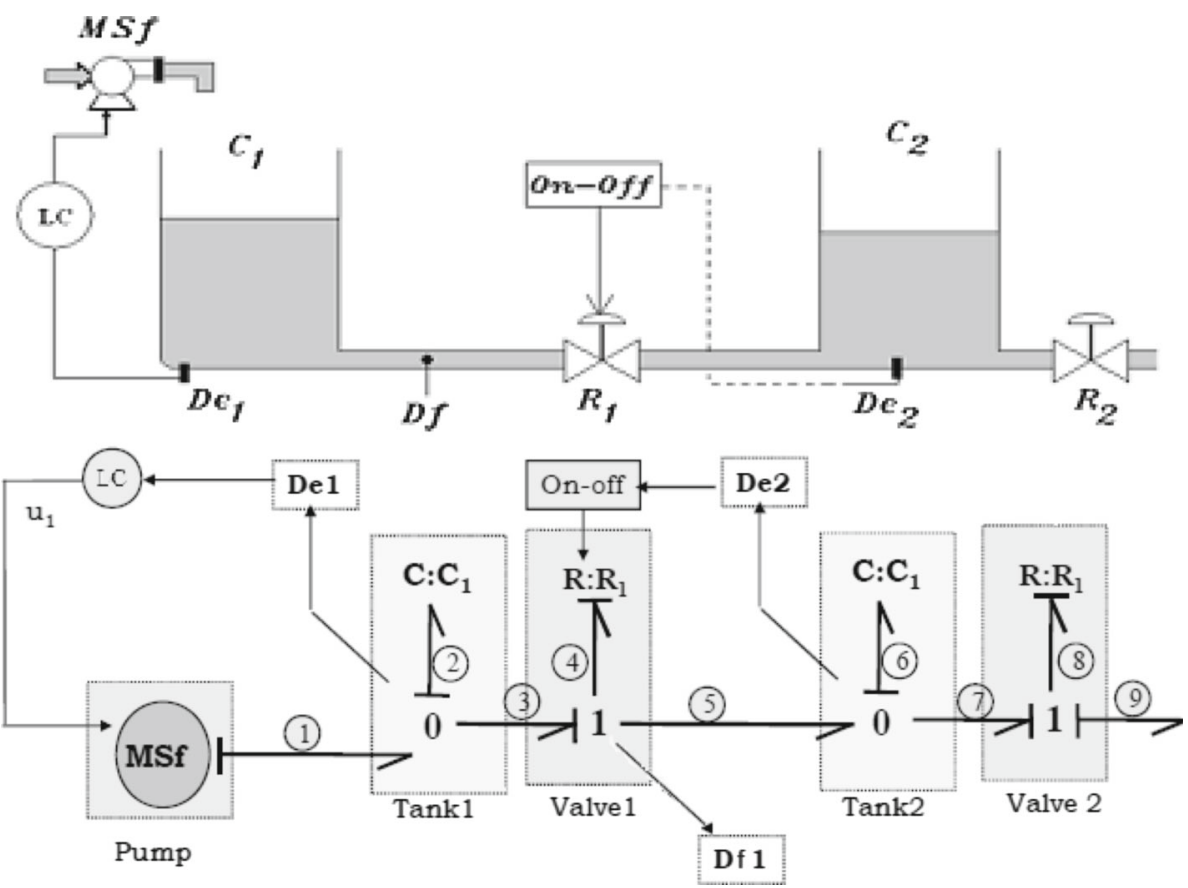

Figure 2. Two tanks system and its corresponding BG model. 
Table 1. Faults signature matrix of the two tanks system.

\begin{tabular}{lcccccccc}
\hline & $\mathrm{r}_{1}$ & $\mathrm{r}_{2}$ & $\mathrm{r}_{3}$ & $\mathrm{r}_{4}$ & $\mathrm{r}_{5}$ & $\mathrm{r}_{6}$ & $\mathrm{M}_{b}$ & $\mathrm{l}_{b}$ \\
\hline $\mathrm{CI}$ & 1 & 0 & 0 & 0 & 0 & 0 & 1 & 1 \\
$\mathrm{RI}$ & 0 & 1 & 0 & 0 & 0 & 0 & 1 & 1 \\
$\mathrm{C} 2$ & 0 & 0 & 1 & 0 & 0 & 0 & 1 & 0 \\
$\mathrm{R} 2$ & 0 & 0 & 1 & 0 & 0 & 0 & 1 & 0 \\
MSF & 1 & 0 & 0 & 1 & 0 & 0 & 1 & 1 \\
$\mathrm{u}_{1}$ & 0 & 0 & 0 & 1 & 0 & 1 & 1 & 1 \\
$\mathrm{u}_{2}$ & 0 & 0 & 0 & 0 & 1 & 0 & 1 & 1 \\
De $_{1}$ & 1 & 1 & 0 & 0 & 0 & 1 & 1 & 1 \\
De $_{2}$ & 0 & 1 & 1 & 0 & 1 & 0 & 1 & 1 \\
Df $_{1}$ & 1 & 0 & 1 & 0 & 0 & 0 & 1 & 1 \\
\hline
\end{tabular}

two different signatures, more details on BG fault detection and isolation procedure are given in Benazzouz et al (2009), Samantaray \& Ould Bouamama (2008) and Mellal et al (2011) and references therein. Notice that in this example, elements $\mathrm{C}_{2}$ and $\mathrm{R}_{2}$ have the same faults signatures; therefore we cannot distinguish which one had caused the fault (Benazzouz et al 2009; Mellal et al 2011). Table 1 shows the faults signature matrix of the system.

To solve the isolability problem between elements, we must add new detectors to the system. Appling our new conversion approach which converts the BG to the Digraph and find the optimal placement of detectors by using only graphical and structural information of the system.

However, in our previous work (Benazzouz et al 2009), we have used the bi-partite graphs to solve the FDI problem. In this part, we present how the Digraph can be used to detect and isolate faults. This is possible because the Digraph illustrates the fault propagation through the system.

To obtain the directed graph of the two tanks system, we have to define the structural adjacency matrix, this matrix is a squared matrix composed by elements of the system in lines and in columns (Alem \& Benazzouz 2011).

If we assume that $\mathrm{G}=(\mathrm{V}, \mathrm{A})$ a digraph, where $\mathrm{V}$ is the vertices and $\mathrm{A}$ is the $\operatorname{arcs}$ of the graph $\mathrm{G}$, thus the structural adjacency matrix of $\mathrm{G}$ is the squared Boolean matrix $\mathrm{M}=\left(\mathrm{m}_{i j}\right)$, of size $\mathrm{n} \times \mathrm{n}$, defined with:

$$
\left\{\begin{array}{c}
m i j=1 \text { if }(i, j) \in A \\
0 \text { else }
\end{array} .\right.
$$

Based on figure 2 and table 1, by selecting the elements and verifying the connection between them we construct the adjacency matrix and the Digraph of the two tanks system. The obtained structural adjacency matrix is given in table 2 . This matrix illustrates the propagation of the information and the flows through the system.

We remark that the structural adjacency matrix is zero-diagonal because there is no circuit between the elements themselves and it is also symmetric. Based on this table, we can construct the Digraph of the two tanks system, knowing that the detectors $\mathrm{D}_{e 1}, \mathrm{D}_{e 2}$ and $\mathrm{D}_{f 1}$ are used to detect the faults on the elements $\mathrm{M}_{s f}, \mathrm{C}_{1}, \mathrm{R}_{1}, \mathrm{C}_{2}$ and $\mathrm{R}_{2}$. Noting that $\mathrm{f}_{1}, \mathrm{f}_{2}, \mathrm{f}_{3}, \mathrm{f}_{4}$ and $\mathrm{f}_{5}$ are the faults that affect the elements $\mathrm{M}_{s f}, \mathrm{C}_{1}, \mathrm{R}_{1}, \mathrm{C}_{2}$ and $\mathrm{R}_{2}$, respectively. Figure 3 represents the obtained Digraph.

This Digraph represents the state equations of the two tanks system by a structural (graphical) representation extracted without need to any analytic formulations. To solve the FDI problem and detect the maximum faults in the system, first we have to add new detectors, because as we can see from figure 3 that $R_{2}$ has no connection to the output. This can also be confirmed in table 2. We propose to add $\mathrm{D}_{f 2}$ as a detector; this will allow the fault $\mathrm{f}_{5}$ that affects the element 
Table 2. Structural adjacency matrix of the two tanks system.

\begin{tabular}{lcccccccc}
\hline & Msf & C1 & R1 & C2 & R2 & De1 & De2 & Df1 \\
\hline Msf & 0 & 1 & 0 & 0 & 0 & 1 & 0 & 0 \\
C1 & 1 & 0 & 1 & 0 & 0 & 1 & 0 & 1 \\
R1 & 0 & 1 & 0 & 1 & 0 & 0 & 1 & 1 \\
C2 & 0 & 0 & 1 & 0 & 1 & 0 & 1 & 0 \\
R2 & 0 & 0 & 0 & 1 & 0 & 0 & 0 & 0 \\
De1 & 1 & 1 & 0 & 0 & 0 & 0 & 0 & 0 \\
De2 & 0 & 0 & 1 & 1 & 0 & 0 & 0 & 0 \\
Df1 & 0 & 1 & 1 & 0 & 0 & 0 & 0 & 0 \\
\hline
\end{tabular}

$\mathrm{R}_{2}$ to be detectable and isolable separately from the fault $\mathrm{f}_{4}$ affected to $\mathrm{C}_{2}$. This problem can be seen in table 1 , where $\mathrm{I}_{b}=0$ for both $\mathrm{C}_{2}$ and $\mathrm{R}_{2}$.

With the additional sensor set, we can detect all the faults on the elements because each one is connected directly to the output. Ms $s_{f}$ represents the modulated pump, since we have eliminated the commands $\mathrm{U}_{1}$ and $\mathrm{U}_{2}$ to simplify the system, thus we are not obliged to add a sensor placement to $\mathrm{Ms}_{f}$. On the other hand, the actuators (inputs) are naturally the known outer vertices in the BG model, whereas the detectors are naturally the unknown outer vertices used to simulate the process behaviour, i.e., in preferred integral causality. In the supervision paradigm, detectors represent measurements from the actual process and hence are imposed as known outer vertices by inverting their causalities.

Now after the conversion of the BG model to the Digraph associated with the linear structured system which is represented by: Fi, the set of faults vertices, Yi, the set of output vertices and

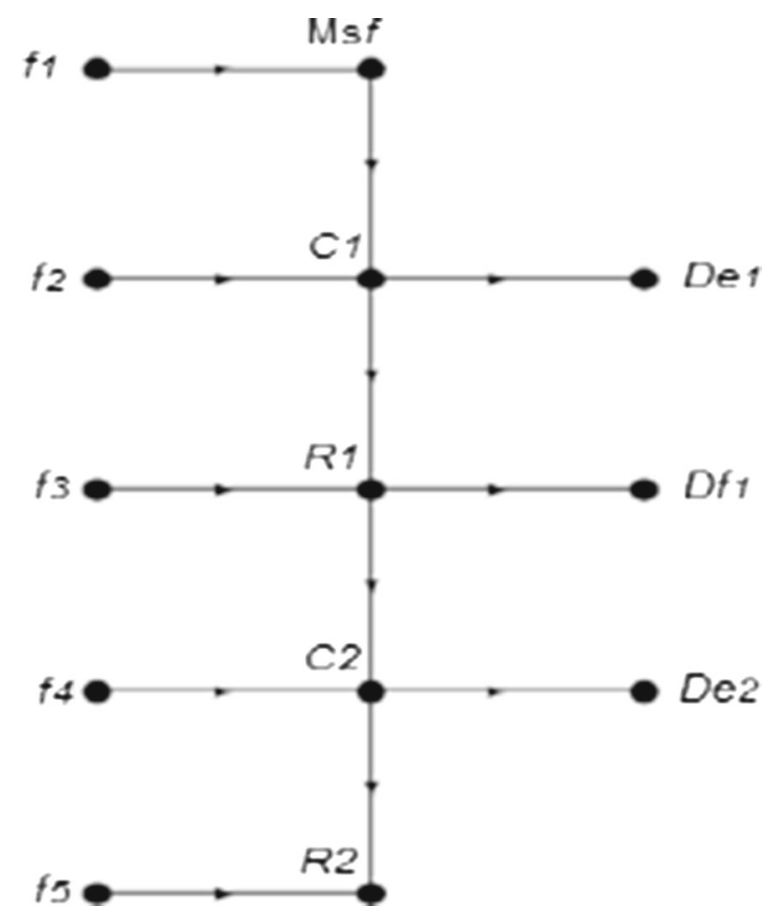

Figure 3. Digraph of two tanks system. 


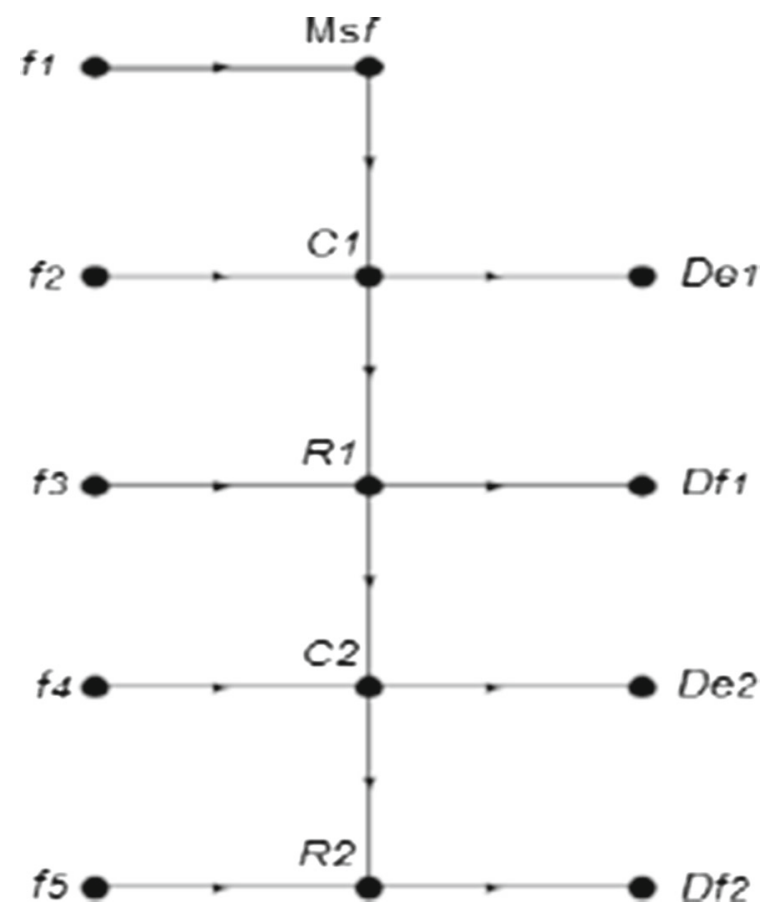

Figure 4. New digraph with optimal sensor placement of two tanks system.

$\mathrm{Xi}$, the set of state vertices (components), we can set-up the optimal sensor placement to detect and isolate all the faults of the two tanks system. The obtained Digraph is given in figure 4. To show the efficiency of this approach, let us consider an industrial application which consists of a water heater.

\section{Application}

In this section, we will detail the conversion method from BG to Digraph based on the novel approach applied to the water heater system. A rather simple example of an industrial water heater system is shown in figure 5, which consists of a thermo-fluid system with localized parameters. It is chosen so, to show the various steps in the conversion from BG to Digraph associating the linear structured system.

Based on figure 5, we construct the corresponding BG model of the system given in figure 6, for more details see Ould Bouamama et al (2006) \& Medjaher et al (2006).

Note that, the level controller (LC) acts upon a pump to maintain a constant level of water in the tank. The water inside the tank is heated by using the thermal energy provided by the heater and the temperature is kept constant at a desired set point by the temperature controller (TC) acting on the thermal source. The valve at the output of the tank is used to deliver a hot water to the consumer. The pump is considered as a flow source, and thus, its output is known. The level (L) and temperature (T) sensors are respectively used to regulate the level and the temperature of the tank. The flow sensor $(\mathrm{F})$ is used to measure the amount of water leaving the tank. The pressure sensor $(\mathrm{P})$ at the bottom of the tank is a material redundancy to $\left(\mathrm{L}=\frac{\mathrm{P}}{\rho \cdot \mathrm{g}}\right)$ the level sensor. 


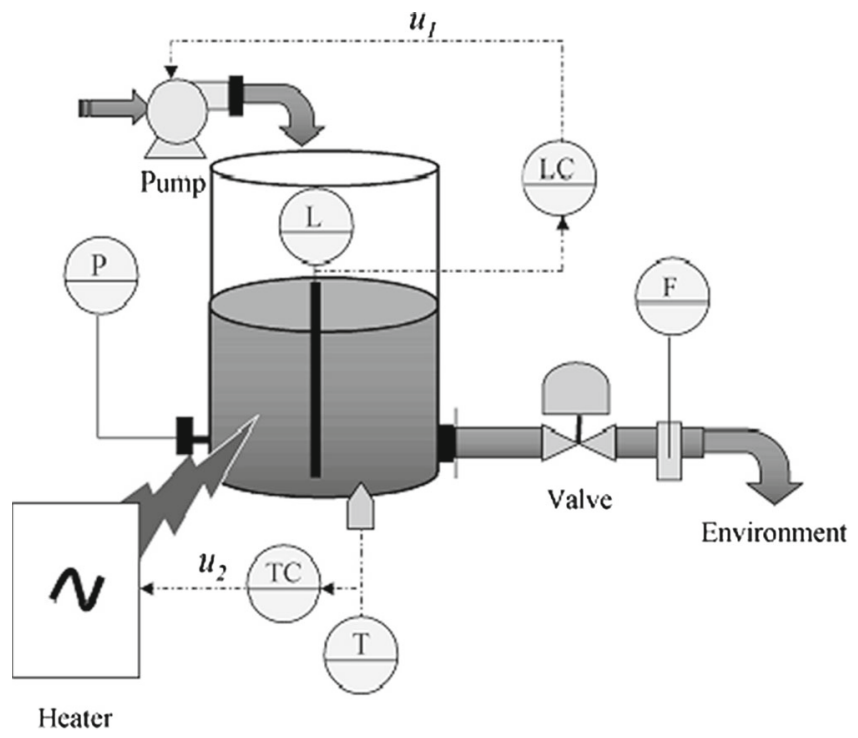

Figure 5. Thermo-fluid system.

In addition, the output control signal of each controller is considered as a known value (Ould Bouamama et al 2006; Medjaher et al 2006).

The theoretical signature matrix of the system shows the fault sensitivity of the residuals derived directly from the analytic redundancy relations ARR and the list of probable faults

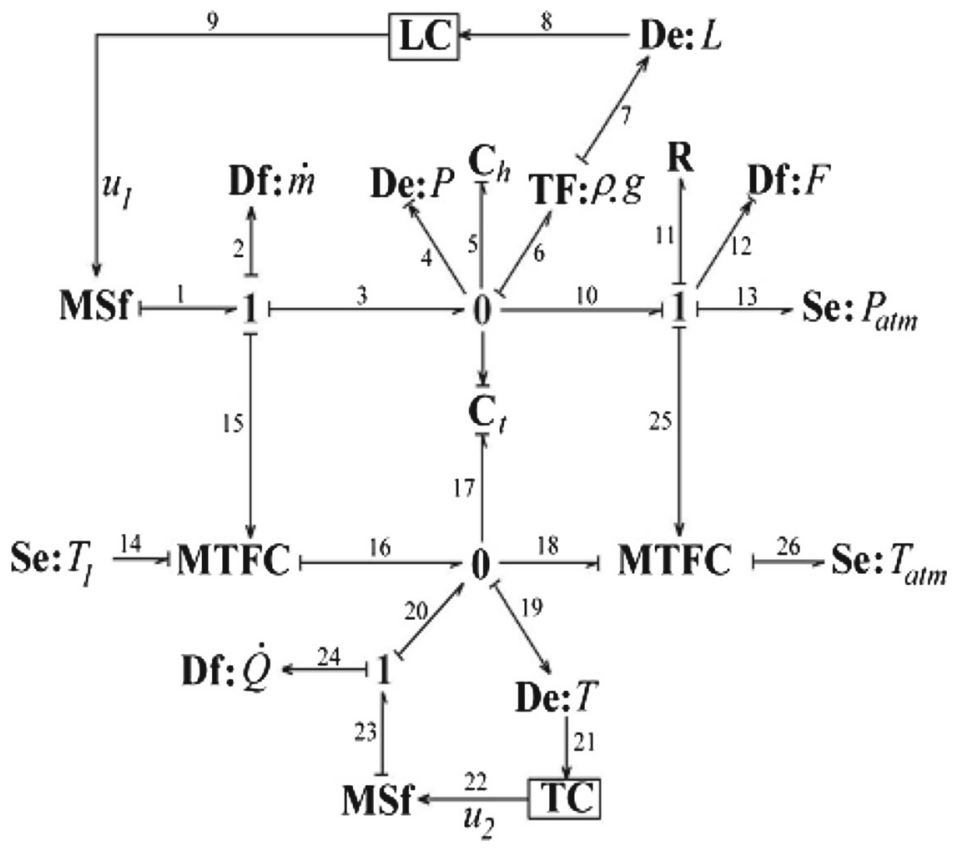

Figure 6. Bond graph model of the thermo-fluid system. 
(Samantaray \& Ould Bouamama 2008), whether they are process, sensor, actuator, or controller failures. To construct this fault signature matrix, we consider that some components are removed from the fault signature since they are supposed to be definitely fault-free. Notice that the environment temperature is never faulty and the controller outputs are correctly measured considering that they are outputs of control software (soft sensors). The obtained fault signature matrix is given in table 3 .

Based on the analysis of this binary matrix, it is found that the signature of each component is present in at least one residual $r_{i}$. All the faults can be detected and isolated theoretically (since each component has a unique fault signature).

Now, to convert the BG model of the system into an associated Digraph, by using the structural adjacency matrix and establish the sensor placement we have to draw the structural adjacency matrix by putting the elements of the system in lines and columns. Then, we check all the connections between elements to fill the Boolean matrix by taking into account following procedures.

(I) To draw the structural adjacency matrix from the BG we have to consider all the elements $\mathrm{R}, \mathrm{C}$ and I as essential components in the matrix because the interconnection between them is represented by the propagation of the flows on the physical system.

(II) Take the detectors De, Df and separate them from the other elements because this will make the sensor placement interpretation simpler. Notice that their interconnections with the other elements are represented by the propagation of the information on the physical system.

(III) The sources and the controllers are the secondary elements in the matrix, thus we can eliminate them. We must put them with the essential elements on the matrix and separate them from the detectors because there is generally an interaction between the detectors and the controllers.

(IV) We must make the same number of elements and it is recommended to not change their positions in line and column of the matrix.

(V) Elements TF and GY are considered to be junctions and we cannot represent them in the matrix because they represent the energy exchange between elements. Generally, if the link between detectors represents information exchange, this means that we have a redundancy in the system.

Table 3. Fault signature matrix of the thermo-fluid system.

\begin{tabular}{lcccccccc}
\hline & $r_{1}$ & $r_{2}$ & $r_{3}$ & $r_{4}$ & $r_{5}$ & $r_{6}$ & $r_{7}$ & $r_{8}$ \\
\hline P (De) & 0 & 0 & 0 & 0 & 0 & 0 & 0 & 1 \\
L (De) & 1 & 1 & 1 & 0 & 0 & 0 & 0 & 1 \\
T (De) & 0 & 0 & 1 & 0 & 0 & 0 & 0 & 0 \\
m (Df) & 0 & 0 & 0 & 0 & 0 & 1 & 0 & 0 \\
Q (Df) & 0 & 0 & 0 & 0 & 0 & 0 & 1 & 0 \\
F (Df) & 1 & 1 & 1 & 0 & 0 & 0 & 0 & 0 \\
Tank & 1 & 0 & 1 & 0 & 0 & 0 & 0 & 0 \\
Valve & 0 & 1 & 0 & 0 & 0 & 0 & 0 & 0 \\
Pump & 1 & 0 & 1 & 0 & 0 & 1 & 0 & 0 \\
Heater & 0 & 0 & 1 & 0 & 0 & 0 & 1 & 0 \\
LC & 0 & 0 & 0 & 1 & 0 & 0 & 0 & 0 \\
TC & 0 & 0 & 0 & 0 & 1 & 0 & 0 & 0 \\
\hline
\end{tabular}


Table 4. Structural adjacency matrix of the thermo-fluid system.

\begin{tabular}{lcccccccccccc}
\hline & $\mathrm{P}(\mathrm{De})$ & $\mathrm{L}(\mathrm{De})$ & $\mathrm{T}(\mathrm{De})$ & $\dot{\mathrm{m}}(\mathrm{Df})$ & $\overline{\mathrm{Q}}(\mathrm{Df})$ & $\mathrm{F}(\mathrm{Df})$ & Tank & Valve & Pump & Heater & $\mathrm{LC}$ & $\mathrm{TC}$ \\
\hline $\mathrm{P}(\mathrm{De})$ & 0 & $\mathbb{1}$ & 0 & 0 & 0 & 0 & 1 & 0 & 0 & 0 & 0 & 0 \\
$\mathrm{~L}(\mathrm{De})$ & 1 & 0 & 0 & 0 & 0 & 0 & 1 & 0 & 0 & 0 & 1 & 0 \\
$\mathrm{~T}(\mathrm{De})$ & 0 & 0 & 0 & 0 & 0 & 0 & 1 & 0 & 0 & 0 & 0 & 1 \\
$\dot{\mathrm{m}}(\mathrm{Df})$ & 0 & 0 & 0 & 0 & 0 & 0 & 1 & 0 & 1 & 0 & 0 & 0 \\
$\overline{\mathrm{Q}}(\mathrm{Df})$ & 0 & 0 & 0 & 0 & 0 & 0 & 1 & 0 & 0 & 1 & 0 & 0 \\
$\mathrm{~F}(\mathrm{Df})$ & 0 & 0 & 0 & 0 & 0 & 0 & 0 & 1 & 0 & 0 & 0 & 0 \\
Tank & 1 & 1 & 1 & 1 & 1 & 0 & 0 & 1 & 1 & 1 & 0 & 0 \\
Valve & 0 & 0 & 0 & 0 & 0 & 1 & 1 & 0 & 0 & 0 & 0 & 0 \\
Pump & 0 & 0 & 0 & 1 & 0 & 0 & 1 & 0 & 0 & 0 & 1 & 0 \\
Heater & 0 & 0 & 0 & 0 & 1 & 0 & 1 & 0 & 0 & 0 & 0 & 1 \\
LC & 0 & 1 & 0 & 0 & 0 & 0 & 0 & 0 & 1 & 0 & 0 & 0 \\
TC & 0 & 0 & 1 & 0 & 0 & 0 & 0 & 0 & 0 & 1 & 0 & 0 \\
\hline
\end{tabular}

By following these procedures, we can construct the structural adjacency matrix of the thermofluid system and convert the BG to the associated Digraph. The obtained matrix is given in table 4. Furthermore, we can make the procedure easier in constructing the structural adjacency matrix by following two steps. First step, directly from the system model given in figure 5 use the detailed instrument plan. Second step, fill the matrix by going through the BG of the model given in figure 6. This will lead to the identical matrix as obtained in the proposed procedure. This approach can be considered in the verification and validation of the BG model. Based on this approach, we construct the structural adjacency matrix (table 4) representing the elements of the thermo-fluid system in lines and columns.

Elements in lines and columns of the table 1 represent the components, actuators and sensors of the thermo-fluid system obtained by analysing its structural model as giving in our pervious work (Alem et al 2013). These elements are given in a Bond Graph context as is given in BG model of figure 2 . Where the Tank component is represented by a BG-C element $\left(\mathrm{C}_{h}\right.$ represents the hydraulic part and $\mathrm{C}_{t}$ the thermal part), the Pump is represented by a BG-Msf element connected to a level controller LC, the Valve with a BG-R element and the Heater with a BG-Msf element connected to a temperature controller TC. Sensors (P, L, T, m, $\bar{Q}, \mathrm{~F})$ are represented with detectors (De, Df).

Moreover, this matrix can be decomposed into 3 sub-matrices given in tables 5, 6 and 7 .

- Structural interconnection sub-matrix (table 5), represents the connection between the essential and secondary elements themselves (R, C, I, sources and controllers in the BG model).

Table 5. Structural interconnection sub-matrix.

\begin{tabular}{lcccccc}
\hline & Tank & Valve & Pump & Heater & LC & TC \\
\hline Tank & 0 & 1 & 1 & 1 & 0 & 0 \\
Valve & 1 & 0 & 0 & 0 & 0 & 0 \\
Pump & 1 & 0 & 0 & 0 & 1 & 0 \\
Heater & 1 & 0 & 0 & 0 & 0 & 1 \\
LC & 0 & 0 & 1 & 0 & 0 & 0 \\
TC & 0 & 0 & 0 & 1 & 0 & 0 \\
\hline
\end{tabular}


Table 6. Structural reachability sub-matrix.

\begin{tabular}{lcccccc}
\hline & Tank & Valve & Pump & Heater & LC & TC \\
\hline P (De) & 1 & 0 & 0 & 0 & 0 & 0 \\
L (De) & 1 & 0 & 0 & 0 & 1 & 0 \\
T (De) & 1 & 0 & 0 & 0 & 0 & 1 \\
m (Df) & 1 & 0 & 1 & 0 & 0 & 0 \\
Q (Df) & 1 & 0 & 0 & 1 & 0 & 0 \\
F (Df) & 0 & 1 & 0 & 0 & 0 & 0 \\
\hline
\end{tabular}

- Structural reachability sub-matrix (table 6), represents the connection between the essential and secondary elements (R, C, I, sources and controllers in the BG model) in first side and the detectors (De, Df in the BG model) in the other side.

This sub-matrix represents the sensor placement of the system, if any component has zero links with any sensor then the observability of its state is impossible thus, we must add a new sensor linking this element, (Commault \& Dion 2003; Alem \& Benazzouz 2011, 2013).

- Structural redundancy sub-matrix (table 7), represents the connection between the sensors/detector themselves. This sub-matrix represents the material redundancy between the sensors. This will help to make an optimal sensor placement and give information redundancy of the system.

Note that in the structural redundancy sub-matrix given in table 7 , there is (1) in the column between the two detectors $\mathrm{P}$ and $\mathrm{L}$ which means that there is a material redundancy between them. This can be confirmed by the $\left(\mathrm{L}=\frac{\mathrm{P}}{\rho \cdot \mathrm{g}}\right)$ equation.

Based on this procedure, we construct the associated digraph of the system, this is illustrated in figure 7.

We can see from the Digraph that the sensor P is connected to the Tank, while the tank is completely connected to other components. With such a configuration, we can deduce that the sensor $\mathrm{P}$ is a redundant element as seen in table 7. In the same manner, the sensor L is an essential sensor for the controller LC as shown in table 6, where $\mathrm{P}$ has no influence on controllers.

However, in this system, we cannot add new sensors. But, we can prove that all faults can be detected and isolated theoretically, since each component has a unique fault signature in the fault signature matrix. The proof of this theoretical aspect was given in our previous work (Alem \& Benazzouz 2013).

Thus, by this approach we can conclude that we have an optimal sensor placement for fault detection and isolation on the thermo-fluid system.

Table 7. Structural redundancy sub-matrix.

\begin{tabular}{lcccccc}
\hline & $\mathrm{P}(\mathrm{De})$ & $\mathrm{L}(\mathrm{De})$ & $\mathrm{T}(\mathrm{De})$ & $\dot{m}(\mathrm{Df})$ & $\bar{Q}(\mathrm{Df})$ & $\mathrm{F}(\mathrm{Df})$ \\
\hline $\mathrm{P}(\mathrm{De})$ & 0 & 1 & 0 & 0 & 0 & 0 \\
$\mathrm{~L}$ (De) & 1 & 0 & 0 & 0 & 0 & 0 \\
$\mathrm{~T}$ (De) & 0 & 0 & 0 & 0 & 0 & 0 \\
$\dot{m}$ (Df) & 0 & 0 & 0 & 0 & 0 & 0 \\
$\bar{Q}$ (Df) & 0 & 0 & 0 & 0 & 0 & 0 \\
$\mathrm{~F}$ (Df) & 0 & 0 & 0 & 0 & 0 & 0 \\
\hline
\end{tabular}




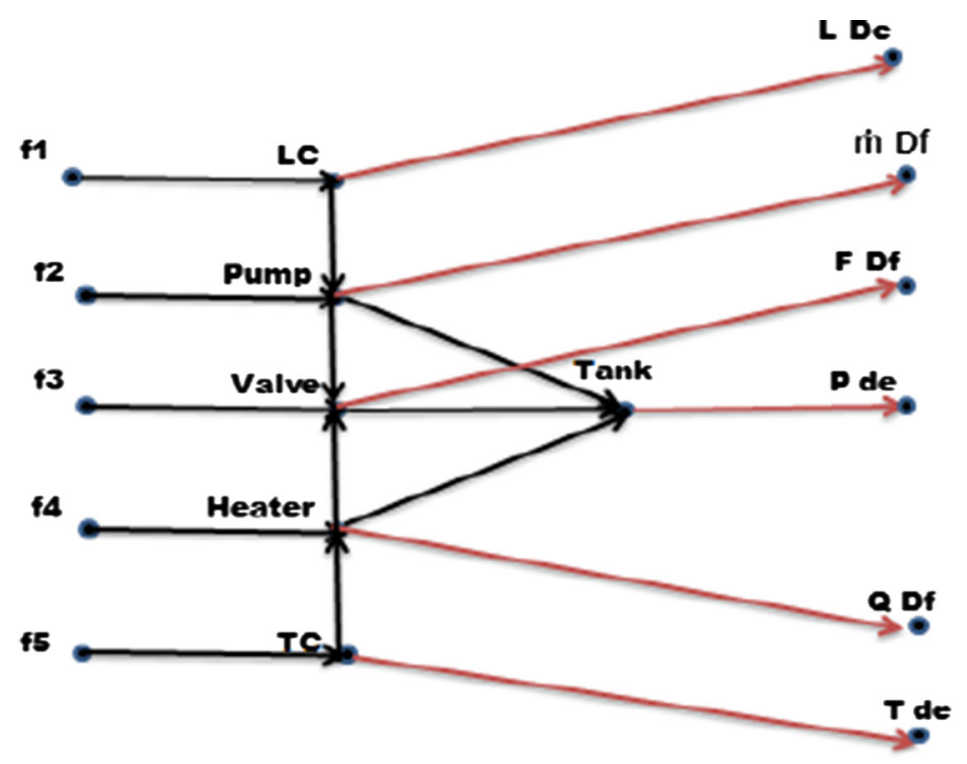

Figure 7. Digraph of the system.

\section{Conclusion}

In this paper, we have considered an FDI problem particularly the problem which has no solution when using classical measurements available on the system. We focused this study on the problem of fault detection and isolation with additional sensors on linear structured systems by using oriented graphs (Digraphs).

We have proposed a novel approach to convert the Bond graph to the Digraph which can be naturally associated with a structured system. This Digraph gives a visual representation of the internal structure and the solvability of several structural problems.

We have defined the structural adjacency matrix, a simple way to construct the Digraph. This matrix is a qualitative approach to solve the FDI problems and the optimal sensors placement on system or components level. This matrix illustrates the propagation of the information and the flows through the system. The structural adjacency matrix is used to validate the Bond Graph model and to optimize the sensor placement on it. This matrix can be decomposed into 3 submatrices that illustrate the reachability and redundancy on sensors and components.

The motivation of BG conversion can be given in two main points. Firstly, this approach can be considered in the verification and validation of the BG model by constructing the structural adjacency matrix by following two steps. First step, directly from the system model given using the detailed instrument plan. Second step, fill the matrix by going through the BG model. This will lead to an identical matrix as obtained with the proposed procedure. Secondly, assuming that the BG model is structurally equivalent to a structured system, where both of them can be formulated in state space form, and therefore each one can be structurally represented as a linear graph where vertices are input, states and outputs and the edges represent the structural inter-connection between the components (variables). That means all structural proprieties and theorems verified on structured systems can be now applied successfully to the converted BG. 


\section{Acknowledgement}

The authors thank the Associate Editors and anonymous reviewers for their valuable suggestions.

\section{References}

Alem S and Benazzouz D 2011 Sensor placement optimization for the isolation of detectable faults, in Proceedings of CMSM'2011, ID174 96 p, Sousse Tunisia

Alem S and Benazzouz D 2013 Optimal sensor placement for fault detection and isolation by the structural adjacency matrix. Int. J. Phys. Sci. 8(6): 225-230. doi: 10.5897/IJPS12.547

Alem S, Benazzouz D and Ichalalen B 2013 Structural analysis for fault detection and isolation using the matching rank algorithm for residual generation: application on an industrial water heating system. Control. Eng. Appl. Inform. 15(2): 20-29

Bell A C and Martens H R 1974 A comparison of linear graphs and bond graphs in the modeling process, Automatic Control Conference

Benazzouz D, Touati Y and Ouled Bouamama B 2009 Dynamic supervision in mechatronic systems using bond graph approach multiphysics 2009 conference, Polyttech'Lille, 9-11 December

Birkett S 1990 Combinatorial analysis of dynamical systems, Thesis, Univ. of Ontario

Blanke M, Kinnaert M, Lunze J and Staroswiecki M 2003 Diagnosis and fault-tolerant control. Springer

Breedveld P 1984 Essential gyrators and equivalence rules for 3-port junction structure. J. Franklin Inst. 318(2): 77-89

Commault C and Dion J M 2003 Optimal sensor location for fault detection and isolation in linear structured systems European Control Conference, ECC 03, 1-4, Cambridge, Angleterre, Sept

Commault C, Dion J M and Perez A 1991 Disturbance rejection for structured systems. IEEE Trans. Automat. Control, 36: 884-887

Dion J M and Commault C 1993 Feedback decoupling of structured systems. IEEE Trans. Automat. Control, 38: 1132-1135

Kamopp D and Rosenberg C 1975 System dynamics: A unified approach. New York: Wiley

König D 1931 Graphak es matrixik. Muter. Fiziol. Lapok. 38116-119

Lin C T 1974 Structural controllability. IEEE Trans. Automat. Control 19: 201-208

Maurya M R, Rengaswamy R and Venkatasubramanian V 2004 Application of signed digraphs-based analysis for fault diagnosis of chemical process flow sheets engineering. Appl. Artif. Intell., 17: 501518

Medjaher K, Samantaray A K, Ould Bouamama B and Staroswiecki M 2006 Supervision of an industrial steamgenerator. Part II: Online implementation. Control. Eng. Pract. 14: 85-96

Mellal Mohamed Arezki et al 2011 Modeling and simulation of mechatronic system to integrated design of supervision: Using a bond graph approach. App. Mech. Mater. 86: 467

Ort R and Martens H R 1974 A topological procedure for converting a bond graph to linear graph. Dynam. Systems Meas. Control. 307-314

Ould-Bouamama B, Medjaher K, Samantaray A and Dauphin-Tanguy G 2005 Model builder using functional and bond graph tools for FDI design. Control. Eng. Pract. (CEP) J. 13: 875-89

Ould Bouamama B, Medjaher K, Samantaray A K and Staroswiecki M 2006 Supervision of an industrial steamgenerator. Part I: Bond graph modelling. Control. Eng. Pract. 14: 71-83

Paynter M 1961 Analysis and design of engineering systems: MIT Press

Perelson A S and Oster G F 1976 Bond graphs and linear graphs. J. Franklin Inst. 302(2): 159-185

Rahmani A, Sueur C and Dauphin-Tanguy C 1997 Approche des Bond Graphs pour I' Analyse Structurelle des Systèmes Linéaires. Linear Algebra Appl. 259: 101-131

Samantaray A K and Ould Bouamama B 2008 Model-based process supervision : A bond graph approach. - (Advances in industrial control) 670.4'27'015115 ISBN-13: 9781848001589, London: Springer Verlag 